\title{
Inhibition of Germinal Centre Apoptotic Programmes by Epstein-Barr Virus
}

\author{
Lindsay C. Spender and Gareth J. Inman \\ Division of Cancer Research, Medical Research Institute, Ninewells Hospital and Medical School, University of Dundee, \\ Dundee DD1 9SY, UK
}

Correspondence should be addressed to Lindsay C.Spender, 1.spender@dundee.ac.uk and

Gareth J. Inman, g.j.inman@dundee.ac.uk

Received 1 June 2011; Accepted 26 July 2011

Academic Editor: Paola Andrea Chabay

Copyright (C) 2011 L. C. Spender and G. J. Inman. This is an open access article distributed under the Creative Commons Attribution License, which permits unrestricted use, distribution, and reproduction in any medium, provided the original work is properly cited.

\begin{abstract}
To establish a persistent latent infection, Epstein-Barr virus (EBV) faces a challenge in that the virus-infected host cell must transit through the germinal centre reaction. This is a site of B cell differentiation where antibody responses are optimised, and the selection criteria for B cells are stringent. The germinal centre environment is harsh, and the vast majority of B cells here die by apoptosis. Only cells receiving adequate survival signals will differentiate fully to be released into the periphery as long-term memory B cells (the site of persistence). In this review, we detail the apoptotic pathways potentially encountered by EBV-infected $\mathrm{B}$ cells during the process of infection, and we describe the functions of those EBV-regulated cellular and viral genes that help promote survival of the host B cell.
\end{abstract}

\section{Introduction}

1.1. The Challenge Faced by EBV to Establish a Latent Infection. Epstein-Barr virus (EBV) persistently infects greater than $90 \%$ of the population, and, in the vast majority of cases, the infection remains benign for life. To establish a persistent latent infection, EBV must access the memory B cell compartment and reside within long-lived peripheral B cells [1] where few viral gene products are expressed in order to escape immune detection. One current model suggests that, to establish latency, EBV transmitted in infected saliva first infects IgD+ve naïve B cells within the tonsils of the nasopharyngeal lymphoid system. EBV-infected cells are then thought to express a limited set of viral genes called the latency III or growth program [2] (see Figure 1). More recent evidence, however, has suggested that transient expression of some lytic cycle genes are also required for the early stages of infection but without eliciting virion production (reviewed in [3]). Following infection, an initial phase of naïve B cell activation and proliferation is driven by viral genes which is orchestrated by the viral transcription factor EBNA-2 [4]. EBNA-2 regulates the transcription of all other latent genes as well as a host of cellular genes including the proto-oncogene c-MYC $[5,6]$. Inadequate cytotoxic T-cell responses at this stage of infection can lead to infectious mononucleosis (IM) which is characterised by expansion of EBNA-2-positive B cells-a pathological condition not evident in normal carriers. In IM, the normal zonal architecture of the germinal centre (GC) is disrupted due to the extensive proliferation of virally infected cells. In this disease state, there is evidence that EBV may infect and drive the proliferation of cells other than naïve cells (including memory and/or (GC) cells) in order to rapidly spread throughout the $\mathrm{B}$ cell population [7]. The extraordinary proliferative capacity of EBV-infected $B$ cells expressing the growth program is evident during in vitro culture since infection of resting or GC B cells results in rapid establishment of continuously proliferating immortalised lymphoblastoid cell lines (LCLs) [8]. Through the expression of the latency III genes, EBV infection may also alter the usual phenotypic characteristics of different $B$ cell subsets [9]. However, studies where normal tonsil tissue sections have been dissected and analysed for EBV status and $\mathrm{B}$ cell phenotype suggest that, in vivo, the expansion of latency III type lymphoblasts is restricted. Instead, the 
EBV-infected cells enter GCs and actively participate in B cell differentiation. During this time, EBV gene expression gradually becomes more limited due to the downregulation of EBNA-2 (reviewed in [10]). EBV-infected cells expressing only the latency II program (EBNA-1, LMP-1, and LMP2) have been detected within GCs, and these infected cells retain phenotypic markers of GC centroblasts and centrocytes $[11,12]$. These studies suggest that latently infected cells have arisen following differentiation of newly infected blasts which transit normally through the GC reaction before finally emerging into the peripheral memory $\mathrm{B}$ cell pool carrying latent episomal viral DNA. Here the virus resides selectively (although not exclusively) within isotypedswitched members of the CD27+ve, IgD-ve memory B cell population $[13,14]$. At this stage, the cells are in what is termed the latency 0 stage, expressing only EBVencoded RNA (EBERs) (Figure 1). It is possible that EBV could access memory B cells via different routes, possibly depending on whether the infection gives rise to the majority of asymptomatic infections or infectious mononucleosis. However, since EBV-infected cells can transit through GCs, it follows that the cells must somehow survive during $\mathrm{B}$ cell differentiation in order to establish latency. The GC is a hostile environment for B cells. The greater proportion of them undergoes apoptosis induced as a result of the elimination of all but those B cells expressing the highest affinity immunoglobulins. In the next sections, we discuss the apoptotic signalling pathways that regulate GC B cells and finally consider the virally encoded gene products that may influence host cell survival and thus establishment of viral latency.

\subsection{The GC Microenvironment}

1.2.1. The GC Reaction. High levels of apoptosis are induced in GCs in order to select effector plasma cells or memory cells capable of expressing high-affinity immunoglobulins of specific subtypes. The process begins with the recognition of antigen by the B cell receptor, along with cognate priming of T-helper cells by antigen presenting dendritic cells. It is generally thought that these activated B cells entering the GC first proliferate rapidly within the "dark zone" (histologically defined as a densely populated area of $\mathrm{Ki} 67^{+\mathrm{ve}} / \mathrm{CD} 77^{\text {+ve }}$ centroblasts). Cells accumulate somatic hypermutations in their immunoglobulin genes which has the advantage of potentially increasing the specificity of the BCR for its antigen (affinity maturation), but, also necessitates the elimination of B cells with autoreactive and low-affinity receptors [15]. The centroblasts differentiate further into nonproliferating centrocytes and migrate to the GC "light zone" which contains follicular dendritic cells (FDCs) and $\mathrm{T}$ cells. Centrocytes carrying the highest affinity, mutated receptors outcompete others for the limiting amounts of foreign antigen displayed on follicular dendritic cells and for the survival signals provided by helper $\mathrm{T}$ cells [16]. In the absence of these survival signals, GC B cells undergo rapid apoptosis. Although the general architecture of the GC is described here, proliferation and apoptosis are not necessarily confined to the dark and light zones, respectively, as cells may traffic between the two regions (reviewed in [17]). In addition, within the light zone, a proportion of centrocytes undergo class switching by DNA recombination to express either $\operatorname{IgG}, \operatorname{IgA}$, or $\operatorname{IgE}$ and alter the function of their immunoglobulins. In summary, several individual signalling pathways activated during the GC reaction (described in more detail below) form an interconnected web of proapoptotic cues which only the most stringent set of survival criteria can overcome.

\subsubsection{Proapoptotic Signals during T-Cell-Dependent B Cell} Responses. Apoptotic pathways, responsible for the elimination of B cells within the GC signal through the TGF- $\beta$ receptor, B cell receptors, and FAS (outlined schematically in Figure 2). Using the Burkitt lymphoma model of GC apoptosis, and comparing cells isolated from tonsil tissue, we have shown previously that autocrine TGF- $\beta$ signalling via the type 1 TGF- $\beta$ receptor ALK5 contributes to the default apoptotic state of normal GC B cells when they fail to secure survival cues from their microenvironment (death by neglect) [18]. TGF- $\beta$ causes cell death independently of the death receptors FAS and TRAIL [19], by inducing the intrinsic apoptosis pathway. Intrinsic apoptosis requires the activation of two members of the BCL-2 family of apoptosis regulators, BAX, and BAK. These proteins reside in mitochondrial membranes and are responsible for regulating membrane permeability, the release of apoptotic factors into the cytoplasm, and ultimately the activation of an initiator of the caspase cascade (caspase 9). As well as regulating GC $\mathrm{B}$ cell homeostasis in the normal GC microenvironment, TGF- $\beta$ signalling is also required for IgA class switching and secretion [20-22]. Proapoptotic signals are also received following the activation of the $\mathrm{B}$ cell antigen receptor (BCR) in the absence of T-cell help. This negative selection process is induced as a result of weak or inappropriate BCR ligation and is critical for eliminating B cells carrying autoreactive or low-affinity $B$ cell receptors which can arise due to somatic hypermutation and class switching of immunoglobulin genes. In this context, signalling through the $\mathrm{B}$ cell receptor, like TGF- $\beta$, induces intrinsic apoptosis. An alternative "extrinsic," FAS-dependent apoptosis pathway also causes spontaneous apoptosis in cells lacking sufficient T-cell help during differentiation.

Coincidentally ligation of the TGF- $\beta$ and BCR receptors results in the induction of the viral lytic cycle by activating the latent-lytic switch gene BZLF-1 [23, 24]. The BZLF-1 promoter contains multiple Smad-binding elements which act in concert to induce gene transcription [25]. Once established, the lytic programme in its own right protects B cells from apoptosis through late gene expression [26], but the function of BZLF-1 is context dependent. Productive viral infection only occurs after BZLF-1 expression when the viral DNA is methylated (i.e., after latent infection is already established) [27].

At what point EBV blocks an apoptotic signalling pathway, whether at the receptor level or downstream at the level of the effector proteins inducing apoptosis, may be determined by the various stages of virus cycle and/or differentiation state of the host cell. The mechanisms of 


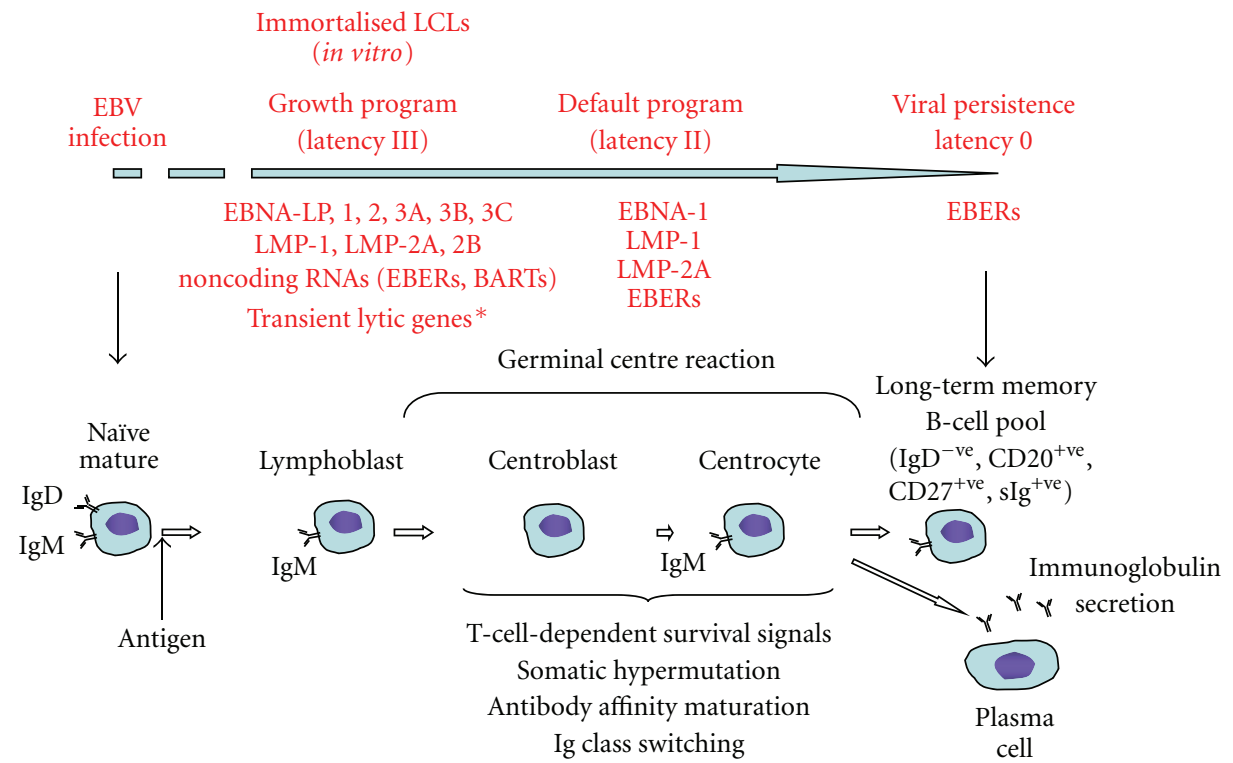

FIGURE 1: Model of establishment of EBV latency in B cells. EBV infects naïe IgD-positive B cells and drives their proliferation by expression of the viral latency III genes, including the latent membrane proteins LMP1, LMP2A, and LMP2B, the EBV nuclear antigens EBNA1, 2, LP, $3 \mathrm{~A}, 3 \mathrm{~B}$, and 3C and noncoding RNA species, the EBV-encoded RNAs (EBERS), and BamHI-A rightward transcripts (BARTs). In vitro, these blasts form continuously proliferating immortalised, lymphoblastoid cell lines (LCLs). EBV-infected cells participate in the GC reaction during which time the number of viral gene products expressed decreases due to the downregulation of the viral transcription factor EBNA2. Following differentiation, long-lived memory B cells emerge as the site of persistent latent infection carrying viral episomal DNA and expressing few viral genes to avoid immune surveillance. ${ }^{*}$ Transient lytic gene expression may occur but without virion production.

apoptosis induction and the various means employed by EBV to abrogate apoptosis are discussed below.

\subsection{Mechanisms of Apoptosis Induction}

1.3.1. TGF- $\beta$ Signalling Pathway in B Cell Apoptosis. TGF$\beta$ signals by enabling the formation of a heterotetrameric complex of the high-affinity type II receptor (T $\beta$ RII) and the type I receptor, ALK5. T $\beta$ RII is a constitutively active serine threonine kinase which, upon receptor complex formation, phosphorylates and activates ALK5, inducing a signalling cascade via the canonical Smad pathway and/or several non-Smad pathways $[28,29]$. TGF- $\beta$ stimulation of ALK5 results in c-terminal phosphorylation of the receptor-regulated Smads, Smad2 (ser465/476) and Smad3 (ser433/435). Following phosphorylation, Smads 2 and 3 bind to the co-Smad, Smad4, and the resulting heteroligoomeric complexes accumulate within the nucleus to both positively and negatively regulate target gene expression [30]. Phosphorylated (activated) Smad2 has been detected by immunohistochemistry within sections of dark and light zones of GC reactions [18]. In centroblasts isolated from GCs, several of the apoptotic genes regulated by TGF- $\beta$ signalling have been characterised and include members of the BCL-2 family acting upstream of BAX and BAK in the intrinsic apoptosis pathway.

The BCL-2 family members which tightly regulate the function of $\mathrm{BAX}$ and $\mathrm{BAK}$ include the prosurvival factors BCL-2, and its homologues, BCL-X $\mathrm{L}, \mathrm{MCL}-1, \mathrm{BFL}-1, \mathrm{BOO}$, and BCL-w. Other members of the family, sharing one region of homology with BCL-2 (BH3-only proteins) (BIK, BID, NOXA, BIM, BAD, HRK, PUMA, and BMF), are proapoptotic. Direct "activators" of BAX and BAK [31] include BIM, tBID (the active, truncated form of BID), and PUMA [31-33] which activate BAX/BAK by direct binding in response to apoptotic stimuli. The prosurvival factors like BCL-2 prevent apoptosis by sequestering these "activator" proteins, but they themselves may be inhibited by interaction with specific $\mathrm{BH} 3$-only proteins [34]. These proteins are often referred to as apoptosis "sensitisers" which may either free activators from the prosurvival factors to enable BAX/BAK activation [35] or, alternatively, block the direct interaction of prosurvival factors with BAX and BAK [36]. The mechanism of action of TGF- $\beta$ in centroblasts and Burkitt's lymphoma lines involves the induction of an apoptotic program via transcriptional upregulation of the proapoptotic $\mathrm{BH} 3$-only proteins PUMA (our unpublished observations) and BIK, while also downregulating the prosurvival factor BCL- $\mathrm{X}_{\mathrm{L}}$. The increase in $\mathrm{BH}$-only proteins and the loss of $\mathrm{BCL}-\mathrm{X}_{\mathrm{L}}$ expression lead to mitochondrial membrane depolarisation and intrinsic apoptosis [18].

1.3.2. Mechanisms of BCR and FAS-Induced Apoptosis. Like TGF- $\beta$, signalling via the BCR causes an increase in BH3only protein expression and induces the intrinsic apoptosis pathway. The proapoptotic proteins BIK and BIM are both induced by BCR signalling. BIM is able to bind all BCL2 prosurvival proteins making it a highly potent inducer of apoptosis and a critical factor in the homeostatic control of $\mathrm{B}$ cells. Its regulation by the BCR is complex involving both 


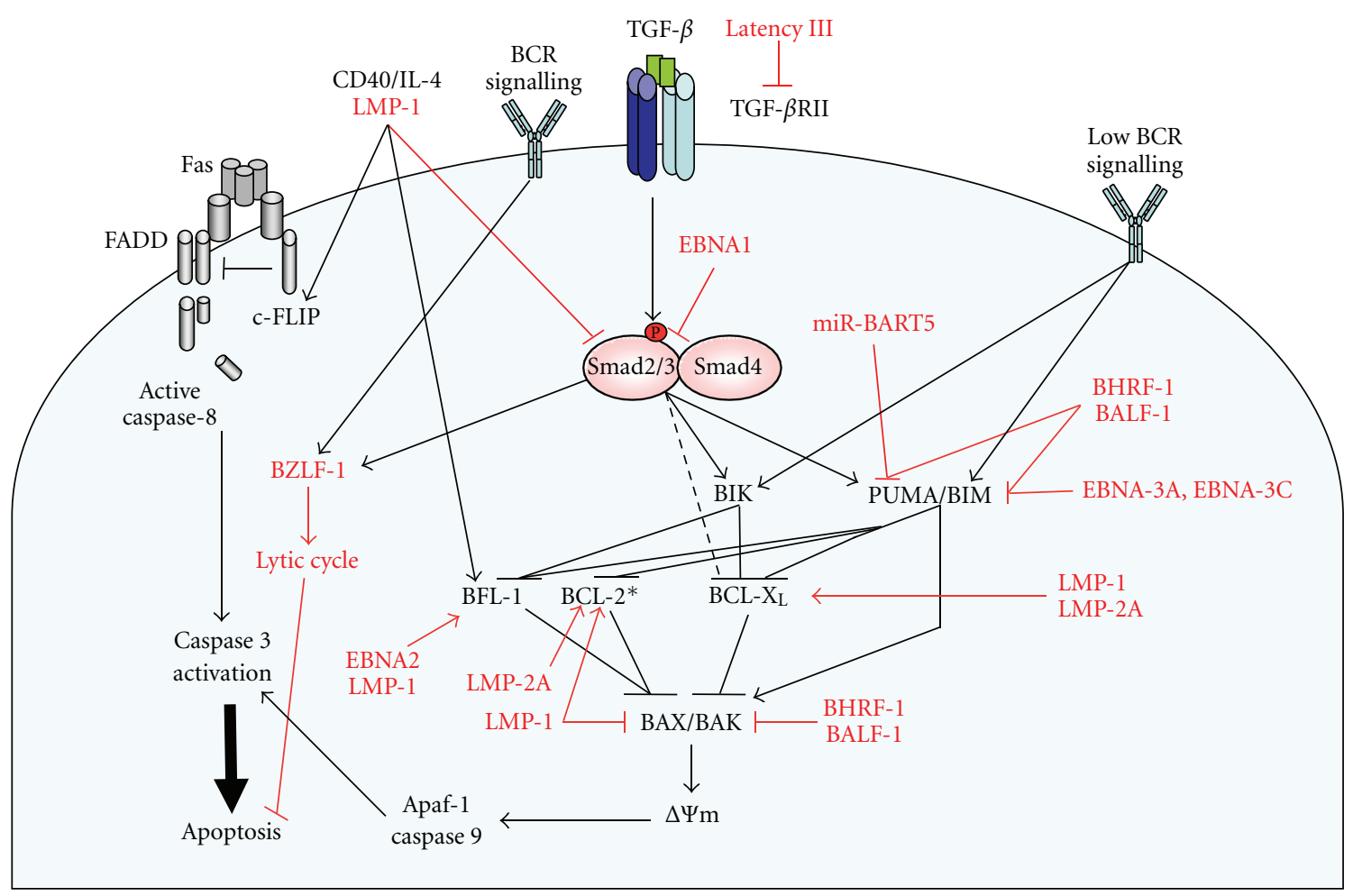

FIGURE 2: EBV infection impacts on cell death and survival pathways in GC B cells. Interconnected signalling pathways regulate the apoptosis of GC B cells. Proapoptotic signals via B cell receptors and the canonical Smad pathway activated by the TGF- $\beta$ receptor control the elimination of unwanted B cells by inducing intrinsic apoptosis. Intrinsic apoptosis is dependent on the activation of the proapoptotic BCL-2 family members BAX and BAK, permeabilisation of the mitochondrial membrane $(\Delta \Psi m)$ and release of proapoptotic factors resulting in the activation of the initiator caspase, caspase 9. An extrinsic apoptotic pathway occurs via the death receptor FAS. FAS stimulation results in the formation of the death-inducing signalling complex (DISC) comprised of FAS, the Fas-associated death domain (FADD), and pro-caspase 8. GC B cells, however, have a preformed DISC whose activation is inhibited by binding of the protein cFLIP. The cells are, therefore, dependent on continuous survival signals via CD40 for maintenance of cFLIP levels. CD40 signalling (along with other signals through BLyS (BAFF) and the BCR not shown on this diagram) also induces BCL-2 family members such as BFL-1 and BCL- $\mathrm{X}_{\mathrm{L}}$ which promote cell survival by inhibiting the intrinsic apoptosis pathway. EBV gene products target multiple points in the apoptosis pathways (shown in red). ${ }^{*}$ In vitro studies of cell lines report the LMP-1-dependent upregulation of BCL-2, however, LMP-1 and BCL-2 expression levels do not correlate in primary tissue.

transcriptional and posttranslational mechanisms (reviewed in [37]). FAS, on the other hand, activates the "extrinsic" apoptosis pathway independent of the BCL-2 family and the mitochondrial response. Unusually, GC B cells have a preformed FAS death-inducing signalling complex (DISC) which lacks any requirement for ligand binding for its activation. Instead, the preformed DISC is held in an inactive form complexed with $\mathrm{CFLIP}_{\mathrm{L}}$. In the absence of adequate GC survival signals from cell-cell contact with $\mathrm{T}$ cells and follicular dendritic cells, cFLIP is degraded, the initiator pro-caspase 8 within the complex is activated, and the cells undergo rapid spontaneous apoptosis [38, 39]. Targeting mitochondrial apoptosis during EBV infection would have the advantage of potentially inhibiting both TGF- $\beta$ and BCRinduced death, whereas FAS-induced apoptosis (which is independent of the BCL-2 family and mitochondria) would need to be targeted selectively.

1.4. Prevention of BCR, FAS, and TGF- $\beta$-Induced Apoptosis by EBV. The viral latent membrane protein LMP-1, which is induced by EBNA-2 and expressed during the growth programme, mimics constitutively active CD40. LMP-1 regulates NF- $\kappa \mathrm{B}$ activity (amongst other signalling pathways) and, in essence, provides the survival signals usually associated with T-cell help. CD40 signalling via NF- $\kappa$ B in GC B cells induces the expression of cFLIP and renders the cells FAS resistant [40]. LMP-1, therefore, provides precisely the signals necessary to counteract FAS-induced apoptosis.

A second mimic of functional B cell receptors, LMP$2 \mathrm{~A}$, is expressed by EBV potentially to inhibit negative selection. EBV LMP-2A functions like its own B cell receptor by constitutively associating with Syk and the Src family of tyrosine kinases [41] normally downstream of BCR signalling. Expressed as part of the growth program, LMP$2 \mathrm{~A}$ is often detected in tumour biopsies of EBV-related malignancies. The effect of encoding its own BCR mimic is that EBV-infected host cells that have lost the capacity to receive normal BCR-derived survival signals (as a result of deleterious or nonsense mutations in the immunoglobulin genes during GC differentiation) can be rescued from 
apoptosis [42, 43]. In vitro infection of CD77-positive (centroblast) GC B cells with EBV can give rise to LCLs with no surface immunoglobulin [44], while LMP2A expression in B cells of BCR-negative mice is sufficient to maintain GC formation in lymphoid tissues of the gut [45]. BCRnegative GC B cells are, therefore, still capable of survival and proliferation by virtue of their positive EBV status. While it is apparent that BCR-negative B cells can survive in vitro and in mouse model systems, there is currently no evidence to suggest that outgrowth of BCR-negative virally infected cells actually occurs in humans in the context of a primary infection with the whole virus. Analysis of immunoglobulin genes expressed within cells of the peripheral memory B cell compartment of IM patients failed to detect any cells expressing defective BCR [14]. In addition, LMP-2A is downregulated during the latency 0 phase in the memory $\mathrm{B}$ cell compartment and could, therefore, have no further role in promoting their survival. It seems likely then that LMP-2A would have a role in augmenting survival in cells with weak BCR signalling within the GC [46] and potentially during tumourigenesis of cells carrying other genetic abnormalities. LMP-2A can also block normal BCR signalling in LCLs that retain BCR expression [47]; however, in nontransformed mouse models of BCR activation, B cell survival appears more dependent on LMP-2A-induced activation of the NF$\kappa \mathrm{B}$ transcription factor and NF- $\kappa \mathrm{B}$ target gene expression than on preventing BCR signalling [48].

EBV-infected cells transiting through GCs within tonsils would undoubtedly encounter TGF- $\beta$ signalling via ALK5. Once immortalised, EBV-infected LCLs are refractory to the inhibitory effects of TGF- $\beta$ signalling. This may, at least in part, be mediated by LMP-1. Treatment of LCLs with antisense to LMP-1 modestly sensitises LCLs to the growth inhibitory effects of TGF- $\beta$ [49] although other studies found no evidence that LMP-1 was either necessary or sufficient to block TGF- $\beta$ responses [50]. In epithelial cells, LMP-1induced activation of NF- $\kappa \mathrm{B}$ interferes with TGF- $\beta$-induced activation of Smad-responsive reporter constructs [51] while in epithelial carcinoma cells and Hodgkin lymphoma cell lines EBNA-1, the viral gene responsible for maintenance and replication of viral episomal DNA, modulates TGF- $\beta$ signalling by reducing Smad2 levels through enhanced protein turnover $[52,53]$. Since the TGF- $\beta$ apoptosis programme is multifactorial, it seems unlikely that one viral protein could be sufficient to disrupt the entire apoptotic response. There are, in fact, numerous points of intersection between apoptosis effectors and EBV-induced survival signals as well as substantial cross-talk between the intrinsic apoptosis pathways of TGF- $\beta$ and the BCR. The viral genes that potentially play a role in blocking both TGF- $\beta$ and BCRinduced apoptosis will therefore be discussed together in the next section.

1.5. Viral Products Disrupting the Expression and Function of Proapoptotic Factors. In some lymphomas associated with EBV infection (Burkitt's lymphoma), insensitivity to TGF- $\beta$ can result from a loss of type II receptor expression $[50,54]$ which correlates with expression of the latency III program although loss of signalling is not mandatory for antagonising the antiproliferative effects of TGF- $\beta$ [55]. This implies that there must be further checks and balances on TGF- $\beta$ target gene expression and/or function to abrogate its effects.

In EBV-infected epithelial cells, the viral latent membrane protein LMP-1 is involved in blocking TGF- $\beta$ mediated antiproliferative effects. LMP-1 can prevent TGF$\beta$-induced cell cycle arrest by suppressing the TGF- $\beta$-induced expression of the transcription factor ATF3. Lack of ATF3 induction enables TGF- $\beta$-mediated expression of Id1 (which would otherwise be inhibited by ATF-3) [56] and the presence of Id1 inhibits TGF- $\beta$-induced cytostasis [57]. In B cells, TGF- $\beta$-induced cytostasis can occur if the apoptotic response is blocked. However, ATF3 does not appear to be a TGF- $\beta$ target gene in B cells, even in the absence of EBV infection. Id 1 protein is upregulated by TGF- $\beta$, but, in B cells, growth arrest can proceed uninterrupted in the presence of Id1 [58]. It, therefore, seems unlikely that LMP-1 regulation of Id1 expression may have a role in interfering with TGF$\beta$-mediated growth arrest in B cells although this has not been tested during infection of resting B cells. Further studies are needed using primary infected material to determine whether LMP-1 expression in the early phase of infection overrides the TGF- $\beta$ cytostatic response in B cells.

1.5.1. Blocking BAX/BAK Function. To potentially combat cell death induced by TGF- $\beta$, EBV employs a variety of prosurvival mechanisms. These include the production of factors which directly counteract the function or induction of the proapoptotic BH3-only proteins (see Figure 2). Several proteins have been implicated in directly blocking the activation of BAX and BAK whose homooligomerization in the mitochondrial membrane [59] is required for apoptosis. In vitro (at least when overexpressed) LMP-1 inhibits BAX promoter activity through the induction of NF- $\kappa \mathrm{B}$ activity [60]. EBV also expresses two viral BCL-2 homologues. The two vBcl-2 genes, BHRF-1 and BALF-1, are maximally expressed just after infection of primary $B$ cells but are not required once latent infection is established in immortalised cell lines. Both genes however, are essential in prevention of spontaneous apoptosis during the earliest stages of infection [61]. Subsequent structural studies have demonstrated that BHRF-1 binds to BAK as well as a subset of BH3-only proteins including the TGF- $\beta$ target gene PUMA, as well as BID and BIM. Interestingly, recombinant BHRF-1 was unable to associate with the "sensitiser" $\mathrm{BH}$-only proteins $\mathrm{BAD}$, BIK, BMF, HRK, or NOXA [62]. BHRF-1, therefore, appears to selectively target all three direct BAX/BAK "activators" which should prove an effective strategy to prevent BAX/BAK activation.

1.5.2. EBV-Mediated Induction of Cellular Prosurvival BCL-2 Family Members. Manipulation of cellular gene expression, such as the prosurvival BCL-2 family members BCL- $\mathrm{X}_{\mathrm{L}}$, BCL-2, MCL-1 and BFL-1, may also contribute to the inhibition of BAX/BAK activation. GC B cells are usually devoid of BCL-2 expression which is transiently downregulated in GC B cells during the transition from naive to 
memory B cell, while BCL- $\mathrm{X}_{\mathrm{L}}$ is induced by CD40 or BCR ligation. Elevated levels of BCL-2 family members in the GC caused by EBV would be expected to provide a survival advantage to infected cells. BFL-1 has been reported to be induced by overexpression of EBNA-2 or LMP-1, through different response elements within the BFL-1 promoter. EBNA-2-dependent transcriptional regulation occurs via a CBF-1/RBP-Jk-binding site [63] while LMP-1-induced expression is dependent on NF- $\kappa$ B activation [64]. LMP-1 is also reported to induce both BCL-2 and MCL-1 expression $[65,66]$, however, more recent studies in vivo using human tissue have found no correlation between LMP-1 expression and expression of BCL-2 [12]. The discrepancy here could be due to the difference between in vitro overexpression studies compared with the function of LMP-1 in vivo in the context of proportionate, and the correct temporal expression of LMP-1.

In vivo experiments using transgenic (LMP2A/HEL-Tg) mice in which B cells express LMP-2A and a specific B cell receptor recognising hen egg lysozyme have now provided useful information regarding the potential role of LMP-2A during BCR activation of EBV-infected cells. There is still the potential caveat that LMP-2A is overexpressed and functions in isolation from potential crosstalk with other EBV latent proteins; nevertheless, this analysis has revealed that LMP-2A mimics BCR survival signals by inducing BCL-2 expression. BCL-2 levels were selectively increased in resting, mature B cells via activation of NK- $\kappa \mathrm{B}$ [48]. In another mouse model, LMP-2A has also been shown to induce BCL- $\mathrm{X}_{\mathrm{L}}$ through constitutive activation of the RAS/PI3K/AKT pathway [67]. The activation of PI3K and elevated BCL- $\mathrm{X}_{\mathrm{L}}$ expression induced by LMP-2A promoted the survival of BCR-negative primary $B$ cells in the periphery (pre-B cell survival signals); however, BCL- $\mathrm{X}_{\mathrm{L}}$ was not increased by LMP-2A in BCRpositive mature $B$ cells in LMP2A/HEL-Tg mice. There are, therefore, potential differences in the effects of EBV genes depending on the differentiation status of the host cell. Further in vivo studies will help determine which members of the BCL-2 family provide essential prosurvival functions and at what stage of EBV infection in vivo.

\subsubsection{Blocking BH3-Only Protein Expression and Function.} Acting at a level above in the hierarchy of BCL-2 family proteins controlling apoptosis, EBV exerts considerable control over the function or expression of the BH3-only proteins. BIK and PUMA are both direct target genes of TGF$\beta$ signalling in B cells. PUMA mRNA is also rapidly and significantly upregulated during spontaneous apoptosis of centroblasts following their isolation and in vitro culture (our unpublished observations). PUMA, like BIM, interacts with all prosurvival factors while BIK selectively inhibits BCL- $\mathrm{X}_{\mathrm{L}}$, BFL-1, or BCL-w [34]. The EBV v-BCL2 homologue BHRF1 directly binds to PUMA (which could potentially interfere with its interaction with other proteins). In addition, EBV encodes its own microRNA (miR-BART5) [68] and induces the cellular microRNA miR-155 [69] which both target PUMA transcripts (discussed below). Over-expression of BHRF-1 has been reported to block apoptosis induced by transient transfection of BIK [70], but it does not appear that the inhibition of BIK function is due to any direct interaction between BIK and BHRF-1 [62]. Instead, the effects of BHRF-1 on BIK function are more than likely due to the inactivation of BAK which, unlike BAX, is required for BIK-mediated apoptosis [71]. Interestingly, BIK also mediates host cell suicide in response to protein synthesis shutoff [71], a process commonly observed following viral infection, and induced by the EBV early lytic gene BGLF5 [72]. BIK is also required for IFN- $\gamma$-induced cell death in human airway epithelial cells [73]. It is possible, therefore, that blocking BIK function may have an important role in evasion of host immune responses.

As well as inhibiting PUMA and BIK function, EBV infection results in a loss of BIM expression $[74,75]$. EBV activation of ERK1/2 kinase, leading to the phosphorylation of BIM and its subsequent degradation by the proteosome, has been proposed as the mechanism for the posttranslational regulation of BIM protein levels [74]; however, other studies suggest that BIM transcription is inhibited through expression of two powerful viral transcriptional repressors EBNA-3A and EBNA-3C [75]. Both viral proteins are essential for immortalisation of primary B cells. EBNA$3 \mathrm{~A}$ and EBNA-3C can cooperate with activated HRas in cell transformation assays and negatively regulate the activity of the viral protein EBNA-2 by competing for binding of the cellular DNA-binding protein RBP-Jk/CBF-1 which is needed to tether EBNA-2 to DNA. In biopsies of EBV-positive Burkitt's lymphomas, the BIM promoter is methylated at CpG dinucleotides suggesting that epigenetic repression of BIM could have an important role in tumourigenesis [76]. There is support for this hypothesis from studies in $\mathrm{E} \mu-\mathrm{M} y \mathrm{c}$ transgenic mice which carry a deregulated $M y c$ transgene under the control of the Ig enhancer region. Deregulation of Myc resembles the chromosomal translocation event associated with the development of Burkitt's lymphoma. In this model, Bim induction, along with the activation of the ARF/p53 pathway, is important for mediating apoptosis caused by the over-expression of Myc. The induction of Puma following Myc-induced p53 activation also performs an important tumour suppressor function in this model which can be overcome, potentially through the induction of Puma binding BCL-2 prosurvival factors downstream of LMP-2A (described above and reviewed in [77]). The loss of a single allele of Bim accelerates the development of the lymphomas demonstrating that Bim also acts as a tumour suppressor in $\mathrm{B}$ cells undergoing oncogenic stress [78]. The case for EBNA-3A and EBNA-3C having the potential to promote EBV-associated malignancy in the face of deregulated MYC expression is clear. It has not yet been demonstrated that this function of the EBNA-3s is required for viral transformation of primary cells, but, given that one of the major cellular targets of EBNA-2 is c-MYC as described earlier, it is entirely plausible that EBNA-3A and EBNA$3 \mathrm{C}$-mediated repression of BIM is essential during the early stages of infection to prevent MYC-induced apoptosis. In addition, both EBNA-3A and 3C are implicated in the joint repression of $\mathrm{p} 16^{\mathrm{INK} 4 \mathrm{a}}[79]$ and $\mathrm{p} 14^{\mathrm{ARF}}$ [80] required for LCL proliferation. Down-regulation of BIM following late gene 
expression may also be required during the viral lytic cycle [81].

1.6. EBV and miRNAs. EBV both regulates the expression of cellular microRNAs as well as encoding 25 of its' own premiRNA's located within noncoding regions of the BHRF1 and BART genes. These ultimately generate four mature BHRF-1 and 40 mature BART miRNAs. microRNAs are small, single stranded noncoding RNAs which block the translation of complementary target mRNA transcripts or, alternatively, result in mRNA degradation. Both of these functions are mediated by an RNA-induced silencing complex [82]. In-depth analysis of the function of EBV-encoded miRNA using recombinant mutant viruses unable to process the longer pre-miRNAs transcripts revealed that, unlike other herpesviruses such as herpes simplex, cytomegalovirus, and Kaposi's sarcoma viruses (which encode microRNAs to help maintain latency), EBV-produced miRNAs have no role in lytic cycle regulation or in maintaining latent infection. Their role primarily seems to involve protecting newly infected primary B cells from spontaneous apoptosis and promoting proliferation during the early phase of infection. Levels of miR-BHRF1-1 and miR-BHRF1-2-3p are four and two-fold higher, respectively, 5 days after-infection compared with expression in established LCLs. Although still expressed in LCL's, they are of lower abundance and appear largely redundant in this context [83]. The cellular targets of the BART miRNAs whose regulation confers a survival advantage on the newly infected cell are difficult to predict and are as yet unidentified.

A number of BART miRNA target transcripts, however, are known and include LMP-1 and the cellular proapoptotic gene PUMA. The majority of studies on BART miRNAs have been carried out in nasopharyngeal carcinoma where BART expression levels are high, so few functional studies have yet been carried out in a B cell background. Targeting LMP-1 with a virally encoded microRNA suggests that the expression levels of LMP-1 are critical and highlights the importance of interpreting over-expression studies with care.

1.7. EBV and p53. Resting primary B cells lack p53 expression and are insensitive to drug-induced DNA damage. Upon infection with EBV, however, p53 levels increase in line with the levels found in B cells stimulated by mitogen. The resultant LCLs are highly sensitive to the activation of p53 by genotoxic agents and undergo apoptosis rather than a cell cycle arrest [84]. Thus, EBV infection per se does not block the upstream signals regulating p53 induction and has no effect on the phosphorylation of p53 in response to DNA cross-linking agents. Latent EBV infection does, however, selectively block the ability of p53 to induce p 21 in response to DNA-damaging agents inducing cross-links and distortions (e.g., cisplatin) rather than double-strand breaks (e.g., etoposide and $\gamma$-irradiation). The outcome of inducing DNA adducts is, therefore, apoptosis rather than p21-mediated cytostasis [85]. There is evidence that EBNA-3C can act as a deubiquitinase which leads to stabilisation of the p53-negative regulator MDM2. The levels of p53 in EBNA-3C over-expressing cells are consequently reduced through p53 degradation [86], although in LCLs, this effect may be diminished by binding of EBNA-LP (EBNA-5) to MDM2 which reportedly blocks its ability to target p53 for degradation [87]. The formation of EBNALP/MDM2/p53 complexes has been proposed to block p53mediated transcription (of p21) and provide an explanation as to how rapidly proliferating LCLs tolerate high levels of wild-type p53 without succumbing to p53-induced cell cycle arrest [87]. During the lytic cycle, BZLF-1 mediates p53 degradation independently of MDM2 function thereby blocking the potential for p53-mediated gene transcription during productive viral infection [88].

\section{Conclusions}

Establishment of a persistent latent EBV infection requires that the infected host cell transits through the GC where networks of proapoptotic signalling pathways execute a rigorous selection procedure over the differentiating B cells. Few $B$ cells survive this process to differentiate fully. To ensure that the EBV-infected host cell is one of them, EBV has at its disposal an array of prosurvival mechanisms which can potentially override external stimuli promoting cell death (such as TGF- $\beta$ and activation-induced apoptosis) as well as protecting the cell from oncogenic stresses induced during EBV-driven cell proliferation. By blocking cell death pathways, many of the EBV-encoded proteins also inadvertently support the accumulation of genetic mutation and thereby promote tumourigenesis. Sustained expression of the latency III programme as in post-transplant lymphoproliferative disease or the more restricted viral gene expression patterns in Burkitt's lymphoma (EBNA-1 and occasionally LMP-2A) and Hodgkin lymphoma (LMP-1, LMP-2, and EBNA-1) are also likely to make tumours differentially dependent on the presence of EBV [89]. It is important to understand when and where EBV proteins might act to prevent apoptosis and in what particular circumstances, (e.g., LMP-2A behaves differently in different situations, inducing expression of BCL$\mathrm{X}_{\mathrm{L}}$ in cells over-expressing Myc [90], but not in normally infected cells). Due to the scarcity of suitable animal models of EBV infection, many of the studies carried out to date have necessarily used established virally infected cell lines or cells over-expressing single viral genes. The results of functional analysis of viral proteins using these systems may at times conflict with the apparent situation in vivo. More detailed analysis of newer animal models and primary human tissue may help resolve some of these discrepancies and aid in the identification of new therapeutic targets in EBV-related diseases.

\section{Acknowledgments}

Research performed in our laboratory described in this review was funded by an Association for International Cancer Research Fellowship and by Cancer Research UK. 


\section{References}

[1] E. M. Miyashita, B. Yang, G. J. Babcock, and D. A. ThorleyLawson, "Identification of the site of Epstein-Barr virus persistence in vivo as a resting B cell," Journal of Virology, vol. 71, no. 7, pp. 4882-4891, 1997.

[2] A. M. Joseph, G. J. Babcock, and D. A. Thorley-Lawson, "Cells expressing the Epstein-Barr virus growth program are present in and restricted to the naive B-cell subset of healthy tonsils," Journal of Virology, vol. 74, no. 21, pp. 9964-9971, 2000.

[3] M. Kalla and W. Hammerschmidt, "Human B cells on their route to latent infection-early but transient expression of lytic genes of Epstein-Barr virus," European Journal of Cell Biology. In press.

[4] A. J. Sinclair, I. Palmero, G. Peters, and P. J. Farrell, "EBNA-2 and EBNA-LP cooperate to cause G0 to G1 transition during immortalization of resting human B lymphocytes by EpsteinBarr virus," The EMBO Journal, vol. 13, no. 14, pp. 3321-3328, 1994.

[5] C. Kaiser, G. Laux, D. Eick, N. Jochner, G. W. Bornkamm, and B. Kempkes, "The proto-oncogene c-myc is a direct target gene of Epstein-Barr virus nuclear antigen 2," Journal of Virology, vol. 73, no. 5, pp. 4481-4484, 1999.

[6] L. C. Spender, G. H. Cornish, B. Rowland, B. Kempkes, and P. J. Farrell, "Direct and indirect regulation of cytokine and cell cycle proteins by EBNA-2 during Epstein-Barr virus infection," Journal of Virology, vol. 75, no. 8, pp. 3537-3546, 2001.

[7] J. Kurth, T. Spieker, J. Wustrow et al., "EBV-infected B cells in infectious mononucleosis: viral strategies for spreading in the B cell compartment and establishing latency," Immunity, vol. 13, no. 4, pp. 485-495, 2000.

[8] J. H. Pope, W. Scott, and D. J. Moss, "Human lymphoid cell transformation by Epstein Barr virus," Nature New Biology, vol. 246, no. 153, pp. 140-141, 1973.

[9] D. Siemer, J. Kurth, S. Lang, G. Lehnerdt, J. Stanelle, and R. Küppers, "EBV transformation overrides gene expression patterns of B cell differentiation stages," Molecular Immunology, vol. 45, no. 11, pp. 3133-3141, 2008.

[10] D. A. Thorley-Lawson, "Epstein-Barr virus: exploiting the immune system," Nature Reviews Immunology, vol. 1, no. 1, pp. 75-82, 2001.

[11] J. E. Roughan and D. A. Thorley-Lawson, "The intersection of Epstein-Barr virus with the germinal center," Journal of Virology, vol. 83, no. 8, pp. 3968-3976, 2009.

[12] J. E. Roughan, C. Torgbor, and D. A. Thorley-Lawson, "Germinal center B cells latently infected with Epstein-Barr virus proliferate extensively but do not increase in number," Journal of Virology, vol. 84, no. 2, pp. 1158-1168, 2010.

[13] T. A. Souza, B. D. Stollar, J. L. Sullivan, K. Luzuriaga, and D. A. Thorley-Lawson, "Influence of EBV on the peripheral blood memory B cell compartment," Journal of Immunology, vol. 179, no. 5, pp. 3153-3160, 2007.

[14] T. A. Souza, B. D. Stollar, J. L. Sullivan, K. Luzuriaga, and D. A. Thorley-Lawson, "Peripheral B cells latently infected with Epstein-Barr virus display molecular hallmarks of classical antigen-selected memory B cells," Proceedings of the National Academy of Sciences of the United States of America, vol. 102, no. 50, pp. 18093-18098, 2005.

[15] I. C. M. MacLennan, "Germinal centers," Annual Review of Immunology, vol. 12, pp. 117-139, 1994.

[16] C. D. C. Allen, T. Okada, H. L. Tang, and J. G. Cyster, "Imaging of germinal center selection events during affinity maturation," Science, vol. 315, no. 5811, pp. 528-531, 2007.
[17] C. D. C. Allen, T. Okada, and J. G. Cyster, "Germinal-center organization and cellular dynamics," Immunity, vol. 27, no. 2, pp. 190-202, 2007.

[18] L. C. Spender, D. I. O’Brien, D. Simpson et al., “TGF- $\beta$ induces apoptosis in human B cells by transcriptional regulation of BIK and BCL-XL," Cell Death and Differentiation, vol. 16, no. 4, pp. 593-602, 2009.

[19] G. J. Inman and M. J. Allday, "Apoptosis induced by TGF- $\beta 1$ in Burkitt's lymphoma cells is caspase 8 dependent but is death receptor independent," Journal of Immunology, vol. 165, no. 5, pp. 2500-2510, 2000.

[20] F. Briere, T. Defrance, B. Vanbervliet et al., "Transforming growth factor beta $(\mathrm{TGF} \beta)$ directs IgA1 and IgA2 switching in human naive B cells," Advances in Experimental Medicine and Biology, vol. 371, pp. 21-26, 1995.

[21] B. B. Cazac and J. Roes, "TGF- $\beta$ receptor controls B cell responsiveness and induction of IgA in vivo," Immunity, vol. 13, no. 4, pp. 443-451, 2000.

[22] S. Borsutzky, B. B. Cazac, J. Roes, and C. A. Guzmán, "TGF$\beta$ receptor signaling is critical for mucosal IgA responses," Journal of Immunology, vol. 173, no. 5, pp. 3305-3309, 2004.

[23] H. Fahmi, C. Cochet, Z. Hmama, P. Opolon, and I. Joab, "Transforming growth factor beta 1 stimulates expression of the Epstein- Barr virus BZLF1 immediate-early gene product ZEBRA by an indirect mechanism which requires the MAPK kinase pathway," Journal of Virology, vol. 74, no. 13, pp. 5810$5818,2000$.

[24] C. L. Liang, J. L. Chen, Y. P. P. Hsu, J. T. Ou, and Y. S. Chang, "Epstein-Barr virus BZLF1 gene is activated by transforming growth factor- $\beta$ through cooperativity of smads and c-Jun/cFos proteins," The Journal of Biological Chemistry, vol. 277, no. 26, pp. 23345-23357, 2002.

[25] T. Iempridee et al., "Transforming growth factor-beta-induced reactivation of epstein-barr virus involves multiple smadbinding elements cooperatively activating expression of the latent-lytic switch BZLF1 gene," The Journal of Virology, vol. 85, no. 15, pp. 7836-7848, 2011.

[26] G. J. Inman, U. K. Binnié, G. A. Parker, P. J. Farrell, and M. J. Allday, "Activators of the Epstein-Barr virus lytic program concomitantly induce apoptosis, but lytic gene expression protects from cell death," Journal of Virology, vol. 75, no. 5, pp. 2400-2410, 2001.

[27] M. Kalla, A. Schmeinck, M. Bergbauer, D. Pich, and W. Hammerschmidt, "AP-1 homolog BZLF1 of Epstein-Barr virus has two essential functions dependent on the epigenetic state of the viral genome," Proceedings of the National Academy of Sciences of the United States of America, vol. 107, no. 2, pp. 850-855, 2010.

[28] Y. Shi and J. Massagué, "Mechanisms of TGF- $\beta$ signaling from cell membrane to the nucleus," Cell, vol. 113 , no. 6, pp. 685700, 2003.

[29] R. Derynck and Y. E. Zhang, "Smad-dependent and Smadindependent pathways in TGF- $\beta$ family signalling," Nature, vol. 425, no. 6958, pp. 577-584, 2003.

[30] J. Massagué, J. Seoane, and D. Wotton, "Smad transcription factors," Genes and Development, vol. 19, no. 23, pp. 27832810, 2005.

[31] A. Letai, M. C. Bassik, L. D. Walensky, M. D. Sorcinelli, S. Weiler, and S. J. Korsmeyer, "Distinct BH3 domains either sensitize or activate mitochondrial apoptosis, serving as prototype cancer therapeutics," Cancer Cell, vol. 2, no. 3, pp. 183-192, 2002.

[32] H. Kim, M. Rafiuddin-Shah, H. C. Tu et al., "Hierarchical regulation of mitochondrion-dependent apoptosis by BCL-2 
subfamilies," Nature Cell Biology, vol. 8, no. 12, pp. 1348-1358, 2006.

[33] T. Gallenne, F. Gautier, L. Oliver et al., "Bax activation by the BH3-only protein Puma promotes cell dependence on antiapoptotic Bcl-2 family members," Journal of Cell Biology, vol. 185, no. 2, pp. 279-290, 2009.

[34] L. Chen, S. N. Willis, A. Wei et al., "Differential targeting of prosurvival Bcl-2 proteins by their $\mathrm{BH} 3$-only ligands allows complementary apoptotic function," Molecular Cell, vol. 17, no. 3, pp. 393-403, 2005.

[35] M. Certo, V. D. G. Moore, M. Nishino et al., "Mitochondria primed by death signals determine cellular addiction to antiapoptotic BCL-2 family members," Cancer Cell, vol. 9, no. 5, pp. 351-365, 2006.

[36] S. N. Willis, J. I. Fletcher, T. Kaufmann et al., "Apoptosis initiated when $\mathrm{BH} 3$ ligands engage multiple Bcl-2 homologs, not Bax or Bak," Science, vol. 315, no. 5813, pp. 856-859, 2007.

[37] L. C. Spender and G. J. Inman, "Targeting the BCL-2 family in malignancies of germinal centre origin," Expert opinion on therapeutic targets, vol. 13, no. 12, pp. 1459-1472, 2009.

[38] A. Hennino, M. Bérard, P. H. Krammer, and T. Defrance, "FLICE-inhibitory protein is a key regulator of germinal center B cell apoptosis," Journal of Experimental Medicine, vol. 193, no. 4, pp. 447-458, 2001.

[39] M. van Eijk, J. P. Medema, and C. de Groot, "Cutting edge: cellular Fas-associated death domain-like IL-1-converting enzyme-inhibitory protein protects germinal center B cells from apoptosis during germinal center reactions," Journal of Immunology, vol. 166, no. 11, pp. 6473-6476, 2001.

[40] M. van Eijk, T. Defrance, A. Hennino, and C. de Groot, "Death-receptor contribution to the germinal-center reaction," Trends in Immunology, vol. 22, no. 12, pp. 677-682, 2001.

[41] C. L. Miller, A. L. Burkhardt, J. H. Lee et al., "Integral membrane protein 2 of Epstein-Barr virus regulates reactivation from latency through dominant negative effects on proteintyrosine kinases," Immunity, vol. 2, no. 2, pp. 155-166, 1995.

[42] R. G. Caldwell, J. B. Wilson, S. J. Anderson, and R. Longnecker, "Epstein-Barr virus LMP2A drives B cell development and survival in the absence of normal B cell receptor signals," Immunity, vol. 9, no. 3, pp. 405-411, 1998.

[43] C. Mancao, M. Altmann, B. Jungnickel, and W. Hammerschmidt, "Rescue of "crippled" germinal center B cells from apoptosis by Epstein-Barr virus," Blood, vol. 106, no. 13, pp. 4339-4344, 2005.

[44] D. Bechtel, J. Kurth, C. Unkel, and R. Küppers, “Transformation of BCR-deficient germinal-center B cells by EBV supports a major role of the virus in the pathogenesis of Hodgkin and posttransplantation lymphomas," Blood, vol. 106, no. 13, pp. 4345-4350, 2005.

[45] S. Casola, K. L. Otipoby, M. Alimzhanov et al., "B cell receptor signal strength determines B cell fate," Nature Immunology, vol. 5, no. 3, pp. 317-327, 2004.

[46] M. A. Swanson-Mungerson, R. G. Caldwell, R. Bultema, and R. Longnecker, "Epstein-Barr virus LMP2A alters in vivo and in vitro models of B-cell anergy, but not deletion, in response to autoantigen," Journal of Virology, vol. 79, no. 12, pp. 73557362, 2005.

[47] C. L. Miller, J. H. Lee, E. Kieff, A. L. Burkhardt, J. B. Bolen, and R. Longnecker, "Epstein-Barr virus protein LMP2A regulates reactivation from latency by negatively regulating tyrosine kinases involved in sIg-mediated signal transduction," Infectious Agents and Disease, vol. 3, no. 2-3, pp. 128-136, 1994.
[48] M. Swanson-Mungerson, R. Bultema, and R. Longnecker, "Epstein-Barr virus LMP2A imposes sensitivity to apoptosis," Journal of General Virology, vol. 91, no. 9, pp. 2197-2202, 2010.

[49] J. L. Kenney, M. E. Guinness, M. Reiss, and J. Lacy, "Antisense to the Epstein-Barr virus (EBV)-encoded latent membrane protein 1 (LMP-1) sensitizes EBV-immortalized B cells to transforming growth factor-beta and chemotherapeutic agents," International Journal of Cancer, vol. 91, no. 1, pp. 8998, 2001.

[50] G. J. Inman and M. J. Allday, "Resistance to TGF- $\beta 1$ correlates with a reduction of TGF- $\beta$ type II receptor expression in Burkitt's lymphoma and Epstein-Barr virus-transformed B lymphoblastoid cell lines," Journal of General Virology, vol. 81, part 6, pp. 1567-1578, 2000.

[51] N. Mori, M. Morishita, T. Tsukazaki, and N. Yamamoto, "Repression of SMAD-dependent transforming growth factor- $\beta$ signaling by Epstein-Barr virus latent membrane protein 1 through nuclear factor- $\kappa \mathrm{B}$," International Journal of Cancer, vol. 105, no. 5, pp. 661-668, 2003.

[52] J. R. Flavell, K. R. N. Baumforth, V. H. J. Wood et al., "Down-regulation of the TGF-beta target gene, PTPRK, by the Epstein-Barr virus-encoded EBNA1 contributes to the growth and survival of Hodgkin lymphoma cells," Blood, vol. 111, no. 1, pp. 292-301, 2008.

[53] V. H. J. Wood, J. D. O’Neil, W. Wei, S. E. Stewart, C. W. Dawson, and L. S. Young, "Epstein-Barr virus-encoded EBNA1 regulates cellular gene transcription and modulates the STAT1 and TGF $\beta$ signaling pathways," Oncogene, vol. 26, no. 28, pp. 4135-4147, 2007.

[54] M. Fukuda, H. Kurosaki, and T. Sairenji, "Loss of functional transforming growth factor (TGF)- $\beta$ type II receptor results in insensitivity to TGF- $\beta$-mediated apoptosis and Epstein-Barr virus reactivation," Journal of Medical Virology, vol. 78, no. 11, pp. 1456-1464, 2006.

[55] M. Horndasch, E. E. Raschke, G. Bommer et al., "Epstein-Barr virus antagonizes the antiproliferative activity of transforming growth factor- $\beta$ but does not abolish its signaling," International Journal of Cancer, vol. 101, no. 5, pp. 442-447, 2002.

[56] Y. Kang, C. R. Chen, and J. Massagué, "A self-enabling TGF $\beta$ response coupled to stress signaling: smad engages stress response factor ATF3 for Id1 repression in epithelial cells," Molecular Cell, vol. 11, no. 4, pp. 915-926, 2003.

[57] A. K. F. Lo, C. W. Dawson, K. W. Lo, Y. Yu, and L. S. Young, "Upregulation of Id1 by Epstein-Barr Virus-encoded LMP1 confers resistance to TGF $\beta$-mediated growth inhibition," Molecular Cancer, vol. 9, article 155, 2010.

[58] L. C. Spender and G. J. Inman, “TGF- $\beta$ induces growth arrest in burkitt lymphoma cells via transcriptional repression of E2F-1," The Journal of Biological Chemistry, vol. 284, no. 3, pp. 1435-1442, 2009.

[59] N. M. George, J. J. D. Evans, and X. Luo, "A three-helix homo-oligomerization domain containing $\mathrm{BH} 3$ and $\mathrm{BH} 1$ is responsible for the apoptotic activity of Bax," Genes and Development, vol. 21, no. 15, pp. 1937-1948, 2007.

[60] T. Grimm, S. Schneider, E. Naschberger et al., "EBV latent membrane protein-1 protects B cells from apoptosis by inhibition of BAX," Blood, vol. 105, no. 8, pp. 3263-3269, 2005.

[61] M. Altmann and W. Hammerschmidt, "Epstein-barr virus provides a new paradigm: a requirement for the immediate inhibition of apoptosis," PLoS Biology, vol. 3, no. 12, article e404, 2005.

[62] M. Kvansakul, A. H. Wei, J. I. Fletcher et al., "Structural basis for apoptosis inhibition by Epstein-Barr virus bhrf1," PLoS Pathogens, vol. 6, no. 12, Article ID e1001236, 2010. 
[63] P. M. Pegman, S. M. Smith, B. N. D’Souza et al., "Epstein-Barr virus nuclear antigen 2 trans-activates the cellular antiapoptotic bfl-1 gene by a CBF1/RBPJ $\kappa$-dependent pathway," Journal of Virology, vol. 80, no. 16, pp. 8133-8144, 2006.

[64] B. D'Souza, M. Rowe, and D. Walls, "The bfl-1 gene is transcriptionally upregulated by the Epstein-Barr virus LMP1, and its expression promotes the survival of a Burkitt's lymphoma cell line," Journal of Virology, vol. 74, no. 14, pp. 6652-6658, 2000.

[65] S. Henderson, M. Rowe, C. Gregory et al., "Induction of bcl2 expression by Epstein-Barr virus latent membrane protein 1 protects infected B cells from programmed cell death," Cell, vol. 65, no. 7, pp. 1107-1115, 1991.

[66] S. Wang, M. Rowe, and E. Lundgren, "Expression of the Epstein Barr virus transforming protein LMP1 causes a rapid and transient stimulation of the Bcl-2 homologue Mcl-1 levels in B- cell lines," Cancer Research, vol. 56, no. 20, pp. 46104613, 1996.

[67] T. Portis and R. Longnecker, "Epstein-Barr virus (EBV) LMP2A mediates B-lymphocyte survival through constitutive activation of the Ras/PI3K/Akt pathway," Oncogene, vol. 23, no. 53, pp. 8619-8628, 2004.

[68] E. Y. W. Choy, K. L. Siu, K. H. Kok et al., "An Epstein-Barr virus-encoded microRNA targets PUMA to promote host cell survival," Journal of Experimental Medicine, vol. 205, no. 11, pp. 2551-2560, 2008.

[69] S. D. Linnstaedt, E. Gottwein, R. L. Skalsky, M. A. Luftig, and B. R. Cullen, "Virally induced cellular microRNA miR155 plays a key role in B-cell immortalization by Epstein-Barr virus," Journal of Virology, vol. 84, no. 22, pp. 11670-11678, 2010.

[70] J. M. Boyd, G. J. Gallo, B. Elangovan et al., "Bik, a novel deathinducing protein shares a distinct sequence motif with Bcl-2 family proteins and interacts with viral and cellular survivalpromoting proteins," Oncogene, vol. 11, no. 9, pp. 1921-1928, 1995.

[71] T. Shimazu, K. Degenhardt, A. Nur-E-Kamal et al., "NBK/BIK antagonizes MCL-1 and BCL-XL and activates BAK-mediated apoptosis in response to protein synthesis inhibition," Genes and Development, vol. 21, no. 8, pp. 929-941, 2007.

[72] M. Rowe, B. Glaunsinger, D. Van Leeuwen et al., "Host shutoff during productive Epstein-Barr virus infection is mediated by BGLF5 and may contribute to immune evasion," Proceedings of the National Academy of Sciences of the United States of America, vol. 104, no. 9, pp. 3366-3371, 2007.

[73] Y. A. Mebratu, B. F. Dickey, C. Evans, and Y. Tesfaigzi, "The $\mathrm{BH} 3$-only protein Bik/Blk/Nbk inhibits nuclear translocation of activated ERK1/2 to mediate IFN $\gamma$-induced cell death," Journal of Cell Biology, vol. 183, no. 3, pp. 429-439, 2008.

[74] C. Clybouw, B. Mchichi, S. Mouhamad et al., "EBV infection of human B lymphocytes leads to down-regulation of bim expression: relationship to resistance to apoptosis," Journal of Immunology, vol. 175, no. 5, pp. 2968-2973, 2005.

[75] E. Anderton, J. Yee, P. Smith, T. Crook, R. E. White, and M. J. Allday, "Two Epstein-Barr virus (EBV) oncoproteins cooperate to repress expression of the proapoptotic tumoursuppressor Bim: clues to the pathogenesis of Burkitt's lymphoma," Oncogene, vol. 27, no. 4, pp. 421-433, 2008.

[76] K. Paschos, P. Smith, E. Anderton, J. M. Middeldorp, R. E. White, and M. J. Allday, "Epstein-Barr virus latency in B cells leads to epigenetic repression and $\mathrm{CpG}$ methylation of the tumour suppressor gene Bim," PLoS Pathogens, vol. 5, no. 6, Article ID e1000492, 2009.
[77] K. T. Bieging, M. Swanson-Mungerson, A. C. Amick, and R. Longnecker, "Epstein-Barr virus in Burkitt's lymphoma: a role for latent membrane protein 2A," Cell Cycle, vol. 9, no. 5, pp. 901-908, 2010.

[78] A. Egle, A. W. Harris, P. Bouillet, and S. Cory, "Bim is a suppressor of Myc-induced mouse B cell leukemia," Proceedings of the National Academy of Sciences of the United States of America, vol. 101, no. 16, pp. 6164-6169, 2004.

[79] L. Skalska, R. E. White, M. Franz, M. Ruhmann, and M. J. Allday, "Epigenetic repression of p16INK4A by latent epsteinbarr virus requires the interaction of EBNA3A and EBNA3C with CtBP," PLoS Pathogens, vol. 6, no. 6, Article ID e1000951, 2010.

[80] S. Maruo, B. Zhao, E. Johannsen, E. Kieff, J. Zou, and K. Takada, "Epstein-Barr virus nuclear antigens $3 \mathrm{C}$ and $3 \mathrm{~A}$ maintain lymphoblastoid cell growth by repressing p16INK4A and p14ARF expression," Proceedings of the National Academy of Sciences of the United States of America, vol. 108, no. 5, pp. 1919-1924, 2011.

[81] L. Oussaief, A. Hippocrate, C. Clybouw et al., "Activation of the lytic program of the Epstein-Barr virus in Burkitt's lymphoma cells leads to a two steps downregulation of expression of the proapoptotic protein BimEL, one of which is EBV-late-gene expression dependent," Virology, vol. 387, no. 1, pp. 41-49, 2009.

[82] G. J. Hannon, “RNA interference," Nature, vol. 418, no. 6894, pp. 244-251, 2002.

[83] E. Seto, A. Moosmann, S. Grömminger, N. Walz, A. Grundhoff, and W. Hammerschmidt, "Micro RNAS of epstein-barr virus promote cell cycle progression and prevent apoptosis of primary human B cells," PLoS Pathogens, vol. 6, no. 8, Article ID e1001063, pp. 69-70, 2010.

[84] M. J. Allday, A. Sinclair, G. Parker, D. H. Crawford, and P. J. Farrell, "Epstein-Barr virus efficiently immortalizes human B cells without neutralizing the function of p53," The EMBO Journal, vol. 14, no. 7, pp. 1382-1391, 1995.

[85] J. O’Nions, A. Turner, R. Craig, and M. J. Allday, “Epstein-Barr virus selectively deregulates DNA damage responses in normal B cells but has no detectable effect on regulation of the tumor suppressor p53," Journal of Virology, vol. 80, no. 24, pp. 1240812413, 2006.

[86] A. Saha, M. Murakami, P. Kumar, B. Bajaj, K. Sims, and E. S. Robertson, "Epstein-Barr virus nuclear antigen 3C augments Mdm2-mediated p53 ubiquitination and degradation by deubiquitinating Mdm2," Journal of Virology, vol. 83, no. 9, pp. 4652-4669, 2009.

[87] E. Kashuba, M. Yurchenko, S. P. Yenamandra et al., "EpsteinBarr virus-encoded EBNA-5 forms trimolecular protein complexes with MDM2 and p53 and inhibits the transactivating function of p53," International Journal of Cancer, vol. 128, no. 4, pp. 817-825, 2011.

[88] Y. Sato, N. Shirata, A. Kudoh et al., "Expression of EpsteinBarr virus BZLF1 immediate-early protein induces p53 degradation independent of MDM2, leading to repression of p53mediated transcription," Virology, vol. 388, no. 1, pp. 204-211, 2009.

[89] D. T. Vereide and B. Sugden, "Lymphomas differ in their dependence on Epstein-Barr virus," Blood, vol. 117, no. 6, pp. 1977-1985, 2011.

[90] R. Bultema, R. Longnecker, and M. Swanson-Mungerson, "Epstein-Barr virus LMP2A accelerates MYC-induced lymphomagenesis," Oncogene, vol. 28, no. 11, pp. 1471-1476, 2009. 


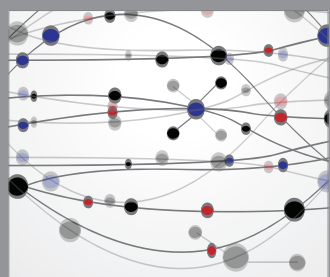

The Scientific World Journal
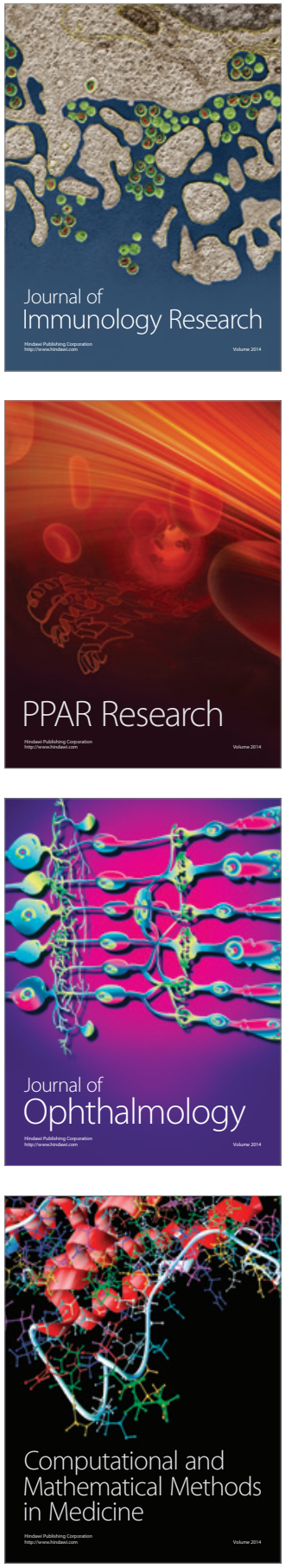

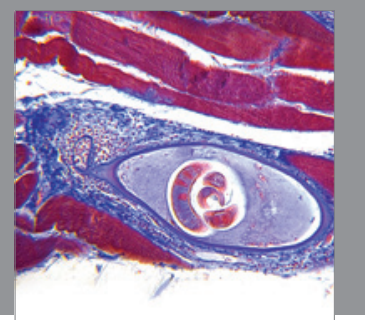

Gastroenterology

Research and Practice
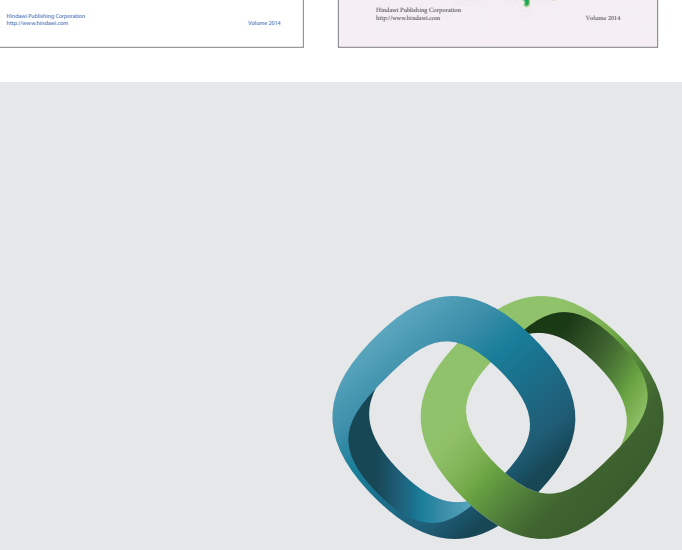

\section{Hindawi}

Submit your manuscripts at

http://www.hindawi.com
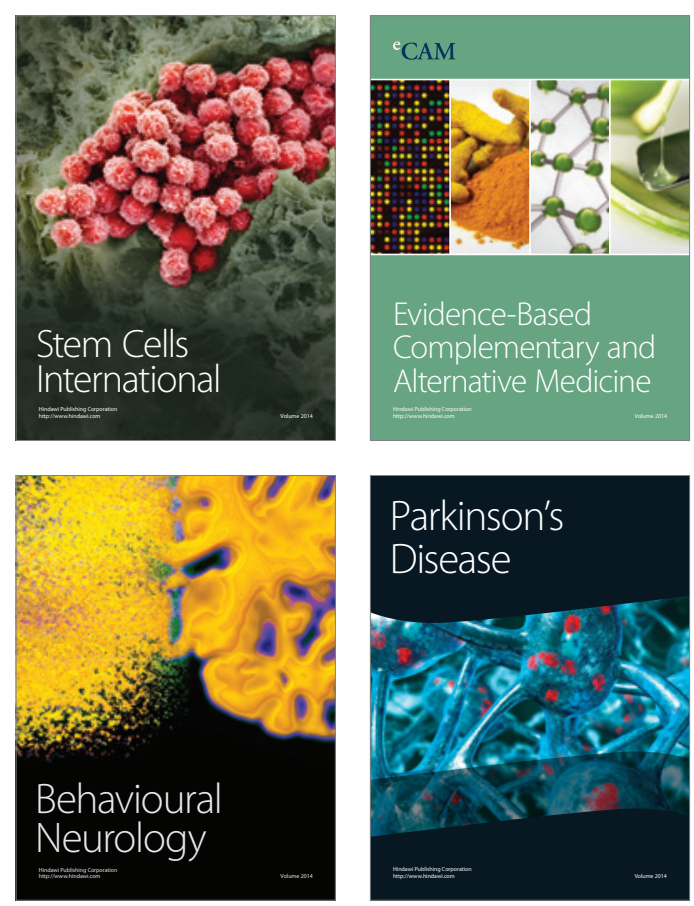

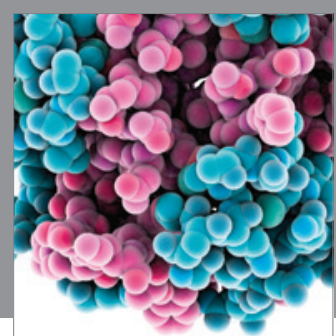

Journal of
Diabetes Research

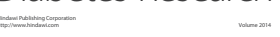

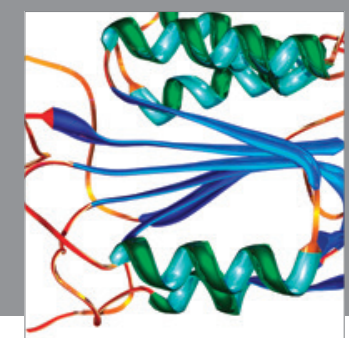

Disease Markers
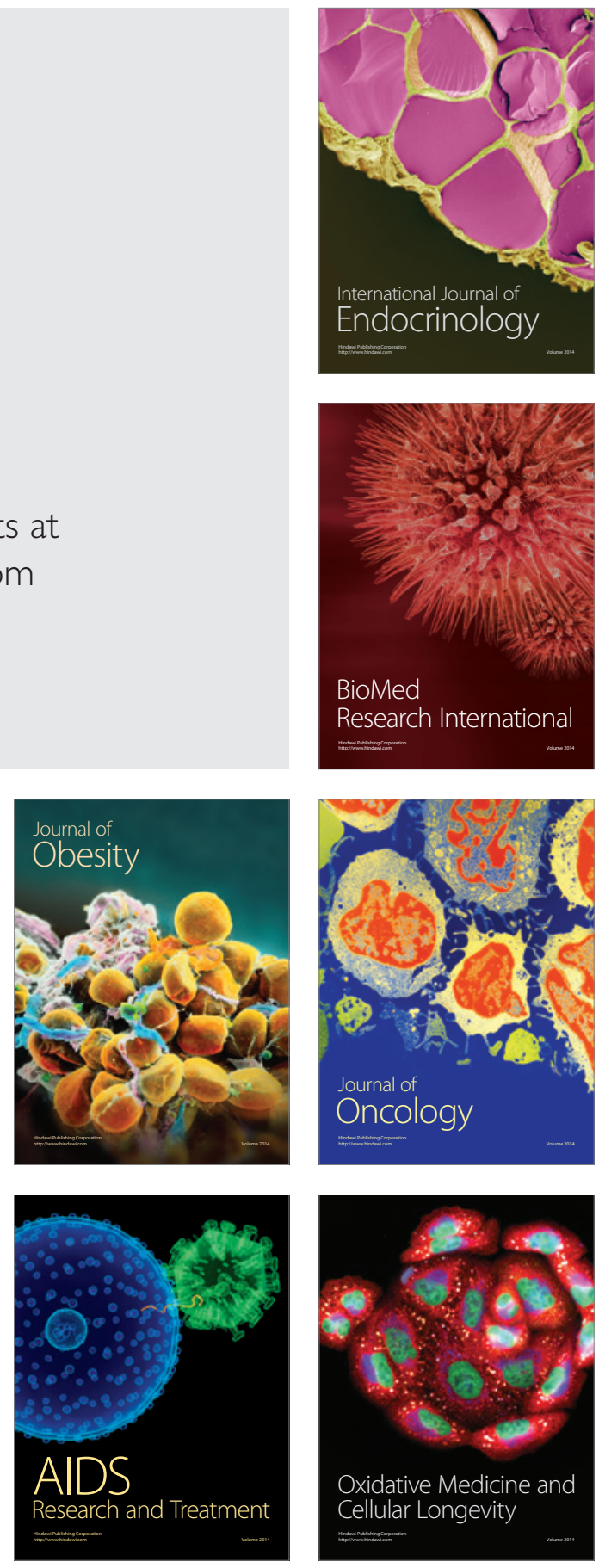